\title{
The Analysis of Effect of Colour Psychology on Environmental Graphic in Children Ward at Medical Centres
}

\author{
Laleh Khodakhah Jeddi ${ }^{1}$, Fereidoon Kasrayee ${ }^{1,2}$, Sanaz Khodakhah Jeddi ${ }^{3}$, \\ Mohammad Taghipour ${ }^{4}$ \\ ${ }^{1}$ Interior Architecture, Non- public Non-profit Institute of Sureh, Tehran, Iran \\ ${ }^{2}$ Field of Interior architecture from France Beaux Arts Décoratifs (Bozar), Tehran, Iran \\ ${ }^{3}$ Architecture Engineering, Islamic Azad University, Yazd Branch, Yazd, Iran \\ ${ }^{4}$ Department of Industrial Engineering, Science \& Research Branch of Islamic Azad Unversity, Tehran, Iran
}

Email address:

laleh.jeddi@gmail.com (L. K. Jeddi), kasrai200@yahoo.com (F. Kasrayee), sanazjeddi@yahoo.com (S. K. Jeddi)

\section{To cite this article:}

Laleh Khodakhah Jeddi, Fereidoon Kasrayee, Sanaz Khodakhah Jeddi, Mohammad Taghipour. The Analysis of Effect of Color Psychology on Environmental Graphic in Children Ward at Medical Centers. Psychology and Behavioral Sciences. Vol. 5, No. 2, 2016 , pp. 51-61. doi: $10.11648 /$ j.pbs.20160502.12

Received: February 17, 2016; Accepted: February 28, 2016; Published: March 29, 2016

\begin{abstract}
The child is a small and very sensitive creature that may react to any stimulant that is created and presented by genetics and environment and need to proper care all the moments, particularly during illness. The child is the beautiful miniature of creation. Unlike our imagination, the child is not the small adult human. This small lively creature differs from an adult person in all dimensions including speech and behavior. Difference among child and an adult human is not restricted to behavioral and verbal issues. But, it is extended to field of disease and therapy as well. For this reason, treatment of child (pediatrics) requires wide psychological and emotional issues., The hospitals are some of architectural spaces, which are assumed as important in terms of design and function due to giving medical services and referrals of various groups of people. Sometimes, compulsory attendance in this space may induce us specific sense where this sense is dubbed along with pain and worry or stress exerted to the patient and this issue may be intensified about children who have mild spirit, weak body, and lower cognition about the environment and this may impose therapeutic process to difficulty and problems for parents of child and personnel. Through analysis in psychological effect of color in environmental graphic (design) by conducting descriptive analysis for exploitation from experiences of these communities toward improvement of climate in national children hospitals, we may come to this result about application of suitable color in graphical design used in this space that what observance of this issue may play basic role in coordination with architectural design space for children hospital in improvement of accepting this climate for children and personnel and reducing some problems such as routing of accelerated activity, reduced stress, mental comfort, and improvement quality of job satisfaction for the given personnel in that space.
\end{abstract}

Keywords: Child, Psychology of Color, Environmental Graphic (Design), Hospital

\section{Introduction}

Application of color is one of therapeutic techniques in complementary and alternative medicine that is called includes noticeable uses for treatment of many physical and spiritual diseases. This technique is one of the oldest natural therapeutic methods in the world along other techniques such as light therapy and stone therapy has been utilized for types of physical and spiritual diseases in various countries of the world such as ancient Egypt, Greece, India, and China.

According to viewpoint of experts, using color medical centers may differently impact on mind and body of humans and employing them properly and or improperly will be followed by positive or negative effects on our life. The therapists and specialists of color-therapy propose unique treatments by the aid of colors in the healthcare field 
particularly soul and mind and creating intellectual and spiritual balance in their patients (Airola Paavo, (2008).

It necessitates knowing comprehensive information about the recovery phases in child, way of caring child, and also role of parents in improvement of child in design of child's ward. As a field that defines spatial quality and environment, the interior architecture design may achieve a proper idea by proper treatment with this issue and considering conditions of users and execute it within an appropriate design.

Considering the psychological effect of color in graphics at Children's war, the present essay tends to create calm and stress-free environment to treat children. Thus, it deals with definitions regarding environmental graphic design and its relationship with child and environmental psychology and their impacts on design of children's ward.

\subsection{Concept of Color}

Isaac Newton, famous English scientist, discovered in 1666 that if the pure white light is passed through a prism it will be synthetized into visible colors. Similarly, Newton discovered that any color included a unique wavelength that might not be synthetized into other colors.

The next experiments showed that one could create various colors by composing lights. For instance, red light is created by composition of yellow and orange lights together. The color which created due to composition of two other colors is called composite color. Some of colors such as yellow and purple will neutralize each other if they are composed and they generate white light. These colors are also called complementary colors (Iten, 2005).

\subsection{Color Physics}

The particulate and wave-like property of light is a physical effect that is felt as a result of light incidence on surface of objects by the eyes. Synthesis of white light by prism is also a physical phenomenon thereby seven colors of the spectrum are created including violet, indigo, blue, green, yellow, orange, and red. Therefore, colors are created by light waves and human's eye can observe the colors that their wavelengths range among 400 to 700 milli-microns (Millimicron is a unit for measurement of waves that is equal to one millionth of a millimeter). In seventeenth century, Newton succeeded to acquire spectrum of lights by synthesis of white light. He discovered that white light included types of rays if they separately hit the human's eye network they would create sense of separate color in eye and mixture of these rays on eye network might make white color sensible (Rodger, 2007).

\subsection{Patient}

Disease is called to abnormality in body or mind that is created due to illness and disorder in function or stress in patient or other related persons with him/ her. In fact, disease is the opposite point to health and with respect to definition of health; disease denotes any type of diversion from physical and mental health that may be overt or covert.
According to definition of World Health Organization (WHO), health denotes full physical, mental, and social comfort and not only lack of disease and or handicapping or disability. Health means enjoying full physical, mental, and social comfort so given that today it is assumed as one of the major human rights all people should have access to the sources needed for providing health (Sayari, 2002).

\subsection{Color Therapy}

Using color (usually as colorful light) for producing therapeutic effects is called color therapy.

The color therapy is not a newly created favorable technique and it has long history. This therapeutic technique have been employed in temples of healing light and color in Heliopolis at ancient Egypt thousand years ago and Before Christ in Greece as well as in ancient cultures in China and India.

Penetration of colors and their impact on human body has been proved by various techniques. Several researches have been conducted about reasons for preference of a certain color to another, effects of colors on human's mind, behavior, sense of intellectual and physical balance, in producing wellbeing or depression, in creating tranquility and or anxiety and some other cases during many years and they have shown the effect of colors in human's life. Also newer investigations have shown during recent years that accurate application of certain colors might be followed by helpful effects on mind health and balance, in physical and mental comfort, in faster retrieval during convalescence period, and in positive stimulation or encouraging retarded children. Colors have various meanings and only looking at each of them cause secretion of certain hormones in body that may lead to comfort, energizing, rising creativity, sound sleeping or reduced anxiety and ours stresses. As a result, color therapy emerged. Human body absorbs the light resulting from chromatic spectra. Any color includes a certain frequency, wavelength, and energy that have been composed with each other. We absorb those colors that could impact on our nervous system, glands, and especially secretion of hormones, and body organism. Doubtlessly, certain colors have economic (sale of products and services), social (selfconfidence and sense of satisfaction or stimulation and encouraging masses of people and many other cases), sexual (stimulation or attraction of opposite sex), mental (happiness or depression), and teaching and training effects (particularly on children) and some other impacts (Stony, 2004).

\section{Methodology}

The descriptive-analytical method has been utilized in this essay and we have employed librarian- field study technique to collect the needed data.

- Searching in specialized journals, CDs, manuals, relevant newspapers and existing books (List of the used resources is proposed at the end of thesis in references and resources part).

- Searching in active and updated Persian and non- 
Persian websites in internet

- Searching in the architectural and non-architectural theses relating to subject of project in universities

- Searching in libraries at public and private organization relating to subject of project including National Iranian Library and Library of Architecture Department in Shahid Beheshti University

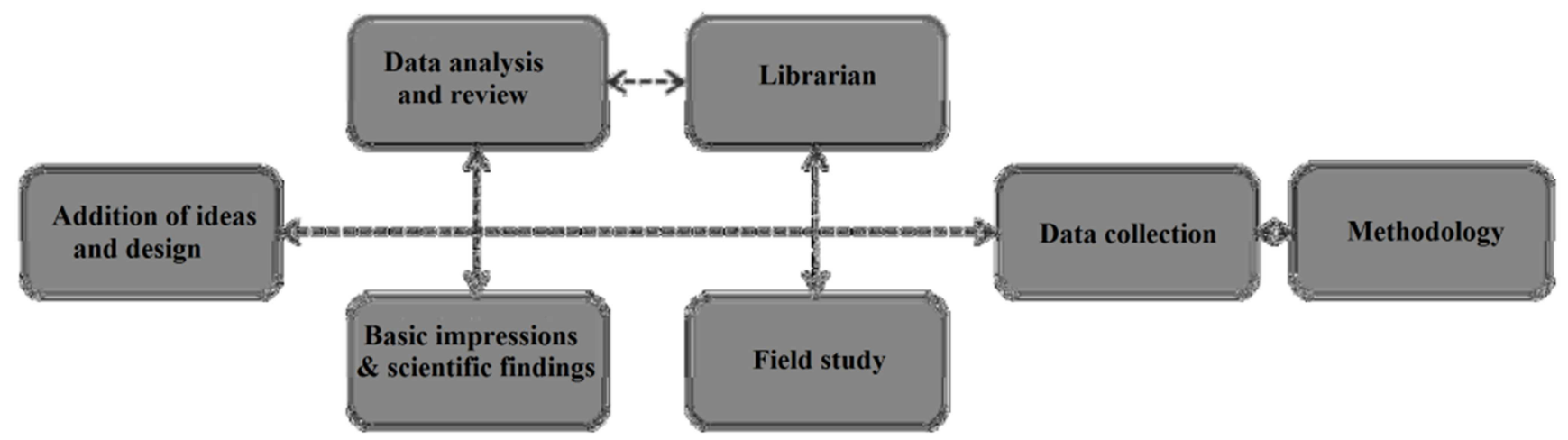

Diagram 1. The suggested methodology (Source: Author).

\subsection{Color Psychology}

Chromotherapy or color therapy is the method of treatment of diseases by means of color. This therapeutic method along with other natural therapeutic techniques such as proper diet, adequate relaxation, exercise, Yuga Asanas and so forth may be employed as an efficient and profitable treatment.

According to comments of chromotherapy clerk (Irola, 2008) the cause of disease may be searched in lack of certain color in body. Chromotherapy treat various diseases by application of colorful light on body. The chromotherapy has been popular therapeutic technique even long times ago. 2500 years ago, Pythagoras used colorful lights for medical purposes and colorful halls were employed for treatment in ancient Egypt, China, and India.

Niels Finsen of Denmark was the pioneer in modern chromotherapy. After discovery of antibacterial effect of solar ultraviolet energy in 1877, Finsen explored the possible treatment of wounds by visible light. Finally, he used red light to prevent from creation of pox wounds and established light institute (it is currently called Finsen's institute and located in Copenhagen, Denmark) in Copenhagen to treat tuberculosis. In 1932, Gerard and Hasey, two Californian psychologists, determined scientifically that blue light had tranquilizing effect and red light had stimulating force in human body (Hosseini, Behshid, 2013).

\subsection{Children-Specific Environmental Graphic Design}

Recognition of spiritual and mental characteristics of children at various age groups may contribute to designer be familiar with their spirit by identifying line and color, space and form and to try for communication with these audiences and to create suitable climate for them. The graphic designer should convey the message for communication with the audiences visually in environmental graphic design and this message is purposive to identify environment. But message and its applied nature is one of dimensions of this subject. One of the other dimensions of this issue is the aesthetic aspect and attempt for beautification of environment and making it favorable. Therefore the graphical forms may transform decoration and beauty and emotional and mental dimension of environment within the other framework in addition to conveying message to observer by presentation of signs and symbols as well and make them pleasant for the audiences (Karimi, Victoria, 2008).

\subsection{Color and Child}

The researchers imagined in the past the child preferred black and white colors to other colors. Such an attitude was developed in 1990s when a manufacturer of toys used black and white colors to make intellectual toys. He made decision based on the studies conducted on perception of colors paradox in baby several years ago. In these experiments, researchers had used chessboard images (black and white) and concluded that black and white colors were more easily recognized than all other colors (These studies were carried out about 1970 i.e. the starting use of computer). But at present it has passed a long time that the researchers know that the children prefer and recognize happy colors since neonatal ages. Various colors may impact on spirit of children and results of studies have shown that children express further interest in blue, red, purple, and orange colors while they are not so inclined to see gray and brown colors (Riazi, Zahra, 2013).

\subsection{Color and Child's Comfort}

All of the existing elements in a room should be addressed for secure and clam environmental design. The similar colors- i.e. those colors are placed beside each other in color cycle- may create clam (Kasebian, 2007). Inter alia, it is suggested using cold colors with low wavelength. The cold colors create a little reduction in body temperature of the observer and cause creating comfortable and thoughtful climate for them so that they are useful for relaxation. Among cold colors, blue color has special position (Mirzakhani, Parviz, 2012). 


\subsection{Effects of Colors in Terms of Psychology}

Whereas effect of colors is relatively subjective and it differs in different persons thus some of color effects possess unique concept throughout the world. The colors locating within spectrum of colors in red zone are characterized as warm colors and this range varies from warm and intimate emotions to sense of anger and wrath.

The colors in range of blue spectrum are called cold colors including blue, purple, and green. These colors are usually comfortable but sometimes may cause sadness and indifference sense in mind.

\subsection{Psychology of Colors as Therapeutic Method}

The color has been utilized in some of ancient cultures including Egyptian's and Chinese. This activity that has been also often called as light therapy or chromotherapy currently is still used alternate therapeutic method.

In this technique:

- Red color is used for stimulation of body and mind and rising concentration.

- Yellow color is employed for stimulation of nerves.

- Blue color is utilized for release pain and ache in patient.

- Indigo color is used for remedy of dermal impairments.

Most of psychologists look at chromotherapy by doubt and suspicion and imply that it has been exaggerated about possible effects of colors and different colors have various concepts in different cultures. The researches have indicated that in most of occasions effects of colors have been transient and temporary impacts in changing moods of individuals. For example, locating persons in a blue room may initially cause sense of comfort in them but this effect may be reduced gradually after they returned to their tranquility (Copper, Maimy, 2014).

\subsection{Psychology of Various Colors}

\subsubsection{Psychology of Black Color}

- Black color absorbs all lights within spectrum of colors.

- Black color is usually used as symbol of fear or Satan but it has been also recognized as sign of power. Black color is utilized to show dangerous characters such as Dracula and or witches.

- Black color is used for mourning ceremonies in most of cultures. Similarly, this color is sign of sadness, sexual attractiveness, and formality.

- Black color was sign of life and repeated birth in ancient Egypt.

- Due to showing body as slim, black color is used in mode and fashion fairs (Davie, 2014)

\subsubsection{Psychology of White Color}

- White color is symbol of innocence and purity.

- White color may create sense of further space in Human.

- White color is usually sign of coldness, cleanness, and tranquility. The room that has been painted by white color may seem spacious and commodious but empty and cold. Hospitals and medical staff use white color for creating sense of cleanness

\subsubsection{Psychology of Red Color}

- Red color is a warm color that stimulates strong emotions.

- Red color represents love, heat, and intimacy.

- Red color creates sense of excitation and feeling.

- Red color stimulates sense of anger and wrath

\subsubsection{Psychology of Blue Color}

- Blue color is the favorable color for most of people and most popular color among males.

- Blue color may create sense of sadness, introversion, and or isolation in some people.

- Blue color is used usually for decoration of offices since the studies have shown that personnel may work more efficiently in blue-colored rooms.

- While blue color is the most popular colors, it is one of the colors which stimulate the least appetite. In some of weight loss programs, it is suggested to put your food on blue dishes. The blue color rarely exists in foods naturally. Similarly, blue color in food is usually considered as sign of contamination and or toxicity of it.

- Blue color may cause tardiness in heart beat and reducing body temperature.

\subsubsection{Psychology of Green Color}

- Green color is a cold color and symbol of nature.

- Green color shows comfort, well-being, health, and jealousy.

- Researchers have found that green color might increase legibility. Some of school and academic students can read and perceive the contents more quickly by putting a transparent green sheet on the book page.

- Green color has been assumed as symbol of fertility since long time ago and in fifteenth century has been used in wedlock cloths.

- Green color is utilized in decoration due to sense of tranquility.

- Green color causes reduction of stress and persons who are working in green workplace may be less subject to pains in alimentary canal.

\subsubsection{Psychology of Yellow Color}

- Yellow color is a warm and happy color.

- Due to higher reflection of light the yellow color makes eyes more tired than most of the rest of other colors. Using yellow color as background of paper or computer display may lead to eye pain or under special conditions to lose sight.

- Yellow color may cause sense of frustration and anger. While yellow color is identified as a happy color, many people may lose their emotion in yellow-colored rooms and also children cry further in yellow rooms.

- Yellow color causes increase in mechanisms of human body. 
- Whereas yellow color is seen earlier than other colors, it is further noticed by persons than other colors.

\subsubsection{Psychology of Purple Color}

- Purple color is symbol of loyalty and wealth.

- Purple color shows wisdom and spirituality.

- Purple color exists very rarely in nature and for this reason it may be considered as sign of artificial or abnormal condition (Davie, 2014).

\subsubsection{Psychology of Brown Color}

- Brown color is a natural color that stimulates sense of power and reliability.

- Brown color also may create sense of sadness and isolation.

- Brown color brings sense of warmth, affection, comfort, and security in mind.

- Brown color usually denotes natural essence, earthliness, and difference but sometimes it can be also sign of complexity.

\subsubsection{Psychology of Orange Color}

- Orange color is composed of red and yellow and considered as an energetic color.

- Orange color brings sense of excitation, warmth, enthusiasm and eager in mind.

- Orange color is usually used to draw their attention.

\subsubsection{Psychology of Pink Color}

- Pink color is in fact the same pale red color and usually symbol of love.

- Pink color has tranquilizing effect. As usual in stadia, the closet room of opponent team is painted in pink color to show their players as less-energetic and passive ones.

Despite of the fact that the tranquilizing effect of pink color has been identified but researchers have found that this impact may only emerge during the initial exposure. For example, when this color was used in prisons, the prisoners became even more uneasy after they were accustomed to it (Child Monthly, 2013).

\subsection{Color in Interior Design of Medical Centers}

Color is one of the latest aspects of interior decorations in hospitals. The designers of modern hospitals have almost deconstructed all of the classic rules regarding the used constraints for use of various colors and their composition together in hospitals and today approximately any color may be utilized in decoration of hospitals.

On the other hand, whereas the classic borders and limits are not observed in application of colors in medical centers therefore this has caused the quality and mechanism for selection of a group of favorable colors to be problematic for decoration of a hospital.

Today, the engineers who construct hospitals and interior designers of small clinics always look for the best strategies based on scientific evidences to use types of colors in their medical centers (Dargahi, Hossein, 2013).
In this section, we intend to show that there are some methods to identify appropriate color composition for any medical ward.

In order to select the most suitable spectrum in painting of medical centers regularly several following scientific and operational factors should be considered:

- Paying attention to psychology of colors and effects of any color on spiritual and physical temperaments of patient and his/her companions

- To notice culture and ethnic spirits and tastes of people at any region

- Preparation of suitable visual platforms for personnel to improve productivity and lack spiritual tiredness in them

- Avoidance from creation of color pollution so that not to lead improper diagnosis of moods in patient

- Analysis of effects of any color in rate of light efficiency and natural and artificial lighting in any space both day and night

- Review of effects of composition in any color spectrum in creating positive and negative errors to diagnose dimensions, distances, magnitude and smallness of any space (Bentley, J, 1997)

The color you select for wall, floor, and ceiling will be surely effective on rate of aesthetics and manifestation of interior space at medical centers. Likewise, it can noticeably impact on feeling of patient, his/ her attendants, and medical personnel of this environment. Further, the selected color may show a room lager, smaller, wider, narrower, higher, brighter, and or warmer.

For example, although a room with bright colored parquets and walls indicates spaces larger, if only bright colors are used the space will be cold and spiritless. This issue will not befit for a medical center that receives patients. Certainly, using various colors one can dub spirit of happiness, hope, and liveliness in hospitals.

Similarly, dimensions and height in most of medical spaces have stark and unpleasant difference from other sites for patients with the existing trend; for example, The narrow and small dimensions of a physiotherapeutic chamber for a patient who should tolerate under meganotherapy for several hours and or magnitude and splendor and vacancy of a radiographic hall in which the patient should spend there alone for some moments may be manifested as transformed by means of suitable composition of various colors and reduce these boring differences for referents to some extent. How can we use properties and effects of colors in medical centers?

Humans' life has been interwoven with colors from the beginning and it can be surely implied that no one could explain about various aspects of human life but without reference to colors and their effects.

The effects of colors are relative of course and they vary in different humans and cultures. But what it does not vary is this important point that no one can deny effects of colors on soul, mind, and even body.

Among them, some group of psychologists looks at effects 
of colors doubtfully since they argue this issue is not directly related to culture of human and it could not be generalized. On the other hand, they assume effects of colors as fleeting and temporary. In response to this issue, it should be declared that even if these effects are temporary, the constructors of hospital could not ignore them.

Even a few moments in reduction of pains and aches of patient and his/ her companion by any healthy means are considered as an achievement for a medical center. In this regard, the hospital construction teams may remarkably contribute to medical teams in these hospitals and provide the backgrounds to return to soul and mind health.
The properties and effects of colors on patients and referents to medical centers are assumed as important and useful if they are not used in a medical center in scientific modern architectural trend in these centers namely oppression to treatment trend in patient and this is an unforgivable mistake.

Whereas today awareness of psychology of colors is deemed as professional requisites for engineers as constructors of hospital thus in necessitates conducting brief review on psychology of colors (Dargahi, Hossein, 2013).

What colors are used in children's hospital?

Table 1. Color and site of use in hospital (Source: Author).

\begin{tabular}{|c|c|c|}
\hline Row & Properties- used site & Image \\
\hline White & $\begin{array}{l}\text { Further and larger space-purity and innocence-suitable for cloths of } \\
\text { physicians and nurses-appropriate for sanitary services }\end{array}$ & \\
\hline Yellow & $\begin{array}{l}\text { Warm color-eyestrain-don't use in nursing stations-a color for drawing } \\
\text { attention-unsuitable for children's space-appetizing color-suitable for } \\
\text { restaurant }\end{array}$ & \\
\hline Orange & $\begin{array}{l}\text { Energetic color-creating excitation-suitable for rising presence of medical } \\
\text { staffs and patient's companions for improving spirit and energy }\end{array}$ & \\
\hline Red & $\begin{array}{l}\text { Heat-happiness-energizing-rising blood pressure and heartbeat-treatment of } \\
\text { rubella and pox in space with red color-suitable for emergency unit and wall- } \\
\text { painting room for child hospitalization }\end{array}$ & \\
\hline Pink & $\begin{array}{l}\text { A type of pale red-symbol of love and comfort-using pink color a little and } \\
\text { in composition with neutral colors is suitable and it is better to be used in } \\
\text { non-medical spaces such as corridors }\end{array}$ & \\
\hline blue & $\begin{array}{l}\text { Symbol of calmness-order- security-reduced heartbeat-anti-appetite-anti- } \\
\text { anxiety-release of nerves-suitable for emergency unit-operation room- } \\
\text { medical wards-room for patient's companions }\end{array}$ & \\
\hline
\end{tabular}




\begin{tabular}{|c|c|c|}
\hline Row & Properties- used site & Image \\
\hline Green & $\begin{array}{l}\text { Symbol of nature-youth-comfort-removal of tiredness-suitable for Special } \\
\text { Care Units in hospital-operation room }\end{array}$ & \\
\hline Brown & $\begin{array}{l}\text { Earthliness-sadness and isolation-power-using in public spaces in hospitals- } \\
\text { provided to composition with happy colors }\end{array}$ & \\
\hline Black & Fear-horror-symbol of sadness-using this color is forbidden in hospital. & \\
\hline
\end{tabular}

\subsection{Several Basic Points About Selection of Colors in Hospitals}

\subsubsection{Paying Attention to Light Effect}

Whether natural and or artificial, light will differently impact on color and knowing this fact is necessary upon design for color in a hospital space. For example, bright yellow walls may seem very aesthetically for a sunny room in tropical and climate and with sunlight but this color will be ugly and boring in walls in a northern rom in which it enjoys lesser natural light.

If colors are executed on surface of walls they will have much strong effect than execution in a piece of paper. When you achieve a satisfactory color composition, pay attention to effect of the existing light in ambience and consider the conditions in the given medical ward as well (training.archnews.ir, 2015).

Please be careful what time of a day and how much natural light radiates on each of your rooms and test your designated color for each of rooms in light of the same room. Composition of colors with each other in a group of wards and effect of the existing light in space in them are the factors that influence in result of work (training.archnews.ir, 2015).

\subsubsection{Paying Attention to Composition of Colors}

There is a coercive point in selection of color for interior spaces in medical centers and that is related to color of various types of medical and hospital equipment and instruments existing inside the given space and they are immoveable. These items include instruments starting from laboratory and or physiotherapeutic equipment in clinics and continue to accessorial instruments of patient's bed and patient's overhead consoles and so forth.

Using colors cycle will be deemed as one of the reliable techniques to find the colors proportional the color of installed equipment in hospital space. Color cycle is composed of twelve colors out of which three colors i.e. red, yellow, and blue are identified as primary colors and the colors among them as secondary colors. If we consider color of medical instruments and equipment in therapeutic sites as the existing colors in this pace we can achieve reasonable results by means of the rest colors from 12 main colors.

Any color in this cycle may be assumed as cognate color with the colors beside it in the same family and also they can be composed well with the opposite color as complementary to create beautiful and harmonic result.

Application of cognate colors from the same family placed beside each other in color cycle and as so-called neighbor colors may create mild and harmonic composition that leads the observer easily to each other.

If application of a color is done properly with correct quantities along with its complementary color it can create bright and eye-catching result and add to attractiveness of the present color composition and avoid from dominance of a certain color over room space (training.archnews.ir, 2015).

\subsubsection{Paying Attention to All Six Dimensions of Space}

We should know that each of six dimensions can impact specifically at its location in medical space. One can invest in each of four sides of walls as well as floor and ceiling in medical spaces. For instance, for a patient who has to see nothing except the ceiling of room for some period of time decoration of ceiling is deemed as the foremost and most valuable point of room and or for a patient who has to stare only at one side or a wall of room that wall will be converted into the paramount physical tool to pacify the soul. Any dimension of space in hospital 
will be deemed as valuable and influential in medical trend of patient (Ibid).

\subsubsection{Paying Attention to Techniques to Create Error in Vision}

One can confuse the persons to recognize real physical nature of medical space through creating a series of conscious and deliberative backgrounds among individuals. This issue may help to reduce abnormalities of medical spaces in patient and his/ her companion and compensate for physical defects. The following techniques may be efficient in this regard:

- If floor and ceiling materials are bright and total walls as dark, room may seem narrower and taller.

- If dark materials were used on floor but colors of walls and ceiling is bright, room space is shown wider.

- If floor and ceiling materials are dark and walls are bright, room space seems to be wider and smaller.

- If ceiling materials are bright but walls and floor are dark sense of locating in basement may be created in patient because light radiates from the top.

- Application of beige and ecru tended to yellow color on floor makes less-lighted and dark places brighter and happier and this color is create comfort.

- Using hazel color on floor will create sense of relationship with earth and nature and will be more beautiful with bright furniture.

- Application of materials with pale beige color on floor creates sense of freshness and cleanness and makes room space brighter.

- Using dark gray materials on floor is neutral, luxurious, and tranquilizing. (Ibid)

\subsection{Color in Children's Hospital}

Florence Nightingale describes diversification in form and brightness in color of the objects shown to patient as a real concept (Dalk et al, 2006). The color may highly impact on perception and response of individuals to the environment and directly influence in improving environmental quality for patients, personnel, and families and rate of recovery of patients so that the studies indicate that the color similar to light may improve rate of treatment up to $10 \%$ (Ibid). Inter alia, what it deemed as important is the comprehensive awareness of designers in using color in reduction of environmental stresses and negative physiologic responses to environmental users. The first point of color application is approaching environment to home space and improvement of symbolic aspect of healing environment that was referred. The positive results are acquired for the patients when natural light and elements, happy colors, pleasant melodies, and controllability of ambience in hospitalization rooms should be considered in designing of hospital (Murphy, 2000; Stickler, 2001). Whereas child may refer to stay in hospital as a concern thus interior design by happy colors may indicate appearance of hospital less formally and create friendly climate for them. A monotonous and boring environment may cause absence of the needed sense for healing. It is because of this fact that brain needs to variation and excitation; The bright white walls which are noticeable in schools cause students to be more serious but the white walls may be unpleasant for most of people and patients because the fantastic nature of them may induce negative sense in patient (Karimi, 2006). According to the conducted observations in hospital, happy colors make child happy by drawing child's attention. Application of suitable colors within room space for hospitalization of children is one of the influential factors on child's spirit in which light plays very important role in its reflection.

\subsection{Psychological Effects of Light}

The color also includes some physiological effects. It has been proved in some experiments that the colorful lights with warm backgrounds cause moving arms and knees toward light while blue and green lights lead to motions at opposite directions.

Many primary school children prevail to color and perceive it. This phase is a transitional period of color and form and it occurs at ages 7-9 years-old; approximately at age 9 through teenage dominancy is more on form than color. When they grow up, display of natural images becomes more important for them. The studies indicate that the small children react to orange color. The pink and orange are some of favorable colors for children. Children express their sensitivity to harmony of colors at age 4 . Although $\mathrm{s} /$ he could not synthetize elements of colors, at ages 8-12, child can synthetize and separate these elements. Such information about selection of artistic works and paintings can be effective for their application in children medical centers (Wills, Pauline, 2013).

\subsection{Applied Suggestions for Painting in Children Ward at Medical Centers}

\subsubsection{Color in Hospitalization Rooms}

Whereas hospitalization room is the most private space in any hospital therefore the personal taste and requirements of the residing patient in this room should be considered in design of decoration and selection of its color more than anything else. Nonetheless, one should not overlook principles of color design in decoration and unconscious impacts left on each of color families in soul and mind of patient.

Rather than considering characteristics of the hospitalized patient in room at any ward, application of medical instruments should be also addressed in the given room. A hospitalization room should be always a tranquilizing and comfortable space to provide appropriate location for sleep and relaxation. It is better for use to avoid from selection of bright color upon design of color in a hospitalization room since such colors may seem annoying during relaxation (Baskaya, 2004: 839-867). 

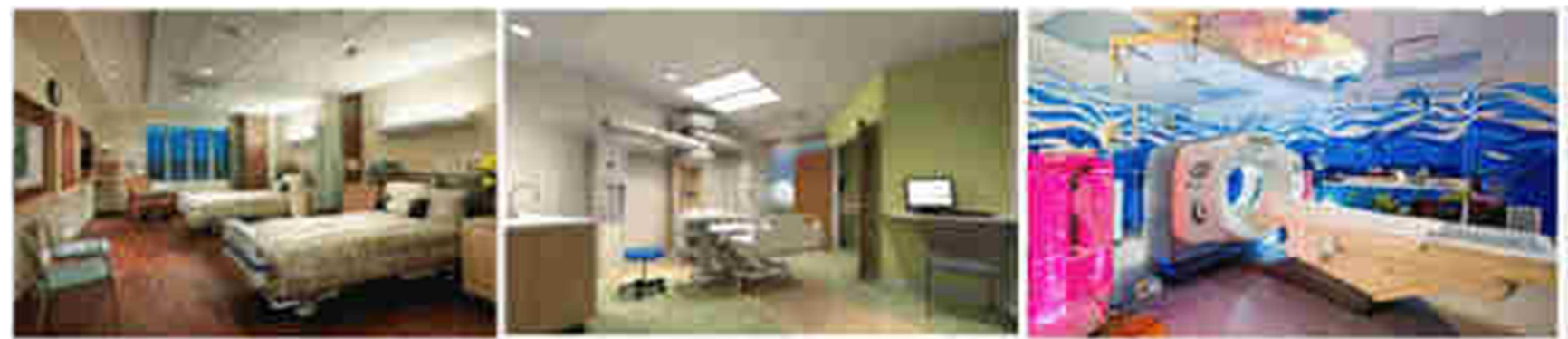

Fig. 1. Application of principles of color in interior graphic design at hospitalization spaces for adults and children (Source: orig09.deviantart.net).

Selection of color in hospitalization room for children includes separate rules. It is better to decorate hospitalization for babies by mild colors. Also, we can embellish hospitalization room for small children and teenagers with happy and more energetic colors.

Using a composition of harmonic colors that are placed along with each other in color cycle in decoration of a hospitalization room in pediatric ward may also be successful. For example, application of different tonalities of violet and green colors along with an opposite bright color such as spatial beige can create beauty. What we should always consider in chromatic design of decoration of a hospitalized room is that this point is crucially important not only in selection of a group of colors but also in quantity of their application and the surface occupied by them in comparison with each other.

\subsubsection{Lobby and Clinic Halls}

If you look for a clinic with warm and a little luxurious space limited application of warm colors such as golden, oranges, and dense reds is the suitable solution for acquisition of such a space. These colors are also deemed as important factor in creation of splendid view on a clinical site.

In addition to employing these colors in clinical spaces, using decorative panels may dub attractiveness of this decoration. To achieve success in composition of surfaces with different designs in such a decoration it requires considering simple and bright surfaces for eye relaxation in the space in which composition of simple and bright colors on walls may play this role well (Gorji Malebani, Yousef, 2013).

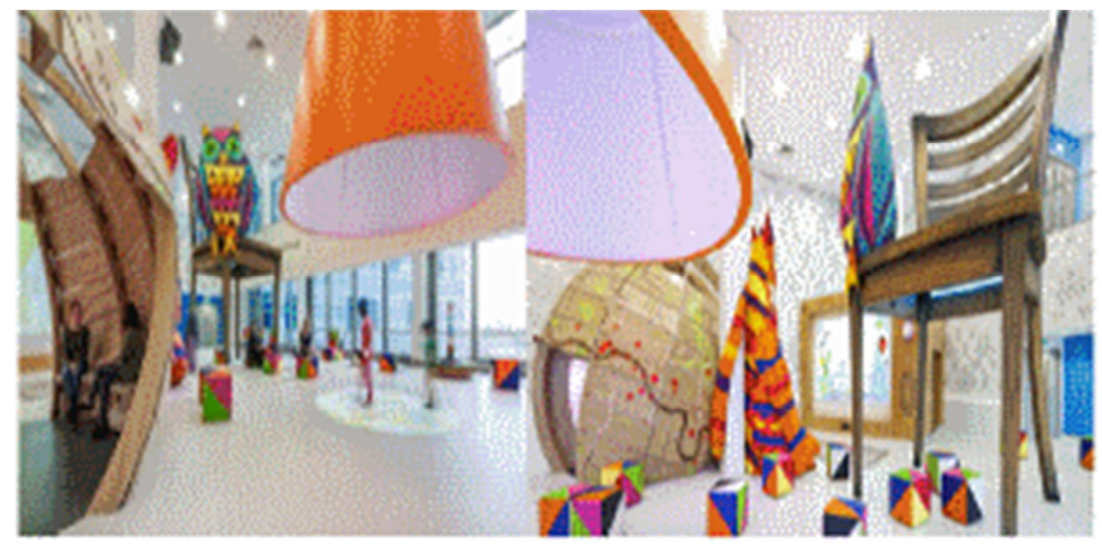

Fig. 2. Application of red-like orange colors and using fascinating elements in environmental graphic designs of lobbies and clinics (Sources: static.gium.co.uk).

In addition to application of these colors in lobby space, using colorful and designed furniture and oriental decorative tableaus and statues may dub attractiveness of this decoration.

\subsubsection{Nursing Station}

In fact, nursing station is the main pole for activities in hospitalization ward. This ward includes different functions such as reception of patients and referents, administrative activities, paging calls, workplace for physicians and nurses particularly during a day. Similarly, computers, information panels, and drug warehouses are located in this ward as well.

Color of this ward may be defined along with color of routing in corridors. Today, it is not recommended to use white color and uniform color in nursing ward that requires concentration and carefulness since the studies have shown that administrative environments by various colors are followed with less stress and error for personnel than administrative environments which are perfectly whitecolored. In fact, quality of design in nursing station directly impacts on improvement of performance of hospital personnel and thus trend of treatment patients. Using comments from nurses and personnel in nursing station may be crucially important in design of this ward since they are more self-aware of the needed equipment and spaces than anyone else. The personnel's attitudes in this ward should be certainly utilized in selection of color for walls of this ward (Gorji Mahlebani, Yousef, 2013). 


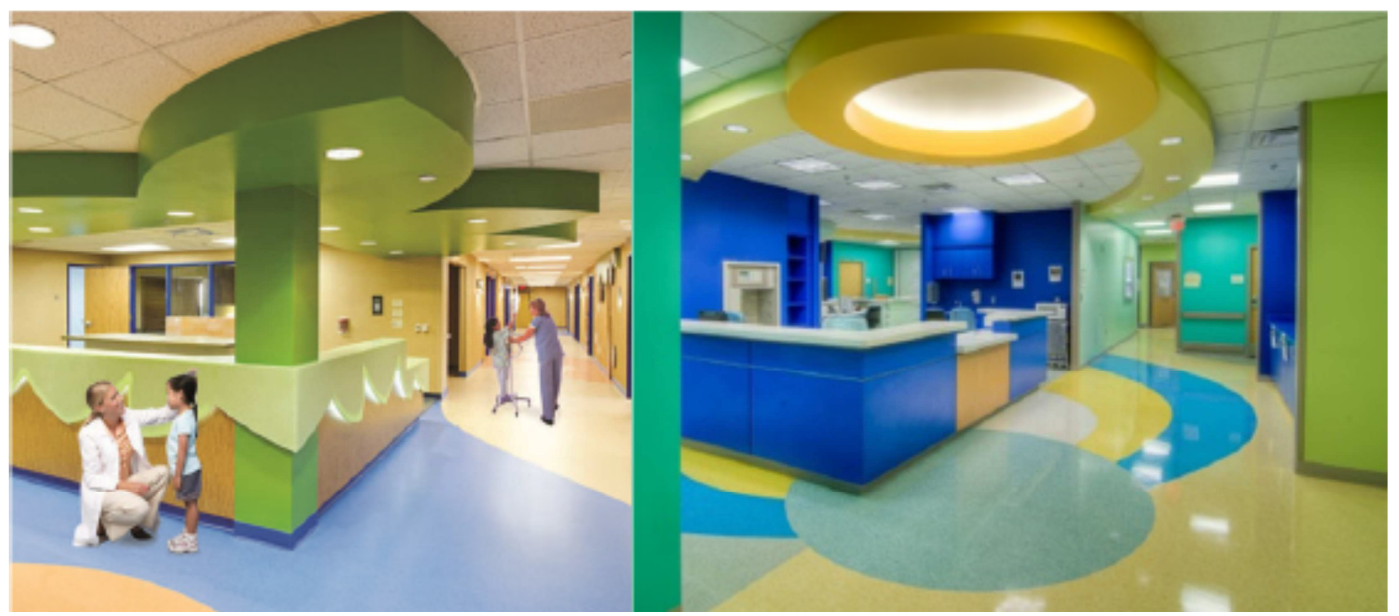

Fig. 3. Application of cold and comfortable colors without eyesore for audience's vision in nursing ward (Source: www.royal-shanghai.com).

The operational level of nursing station should not include excessive color and optical traffic. The findings of researchers indicate that the guiding panels and signs may not be too efficient in an environment that is architecturally and formatively confusing and complex and it may even add to its complexity (Baskaya, 2004).

\subsubsection{Color in Hospitalization of Children}

Using red-herring (distractor) technique in children may divert patients' attention from concentration on pain and fearful processes of treatment and as rate of participation of various senses is further in this process, it will be accompanied by more efficient results. The investigations show that using non- medicinal techniques can be very efficient in reducing pains for children patients (Shamgholi, 2011).

Color of hospitalization environment for children (floor,

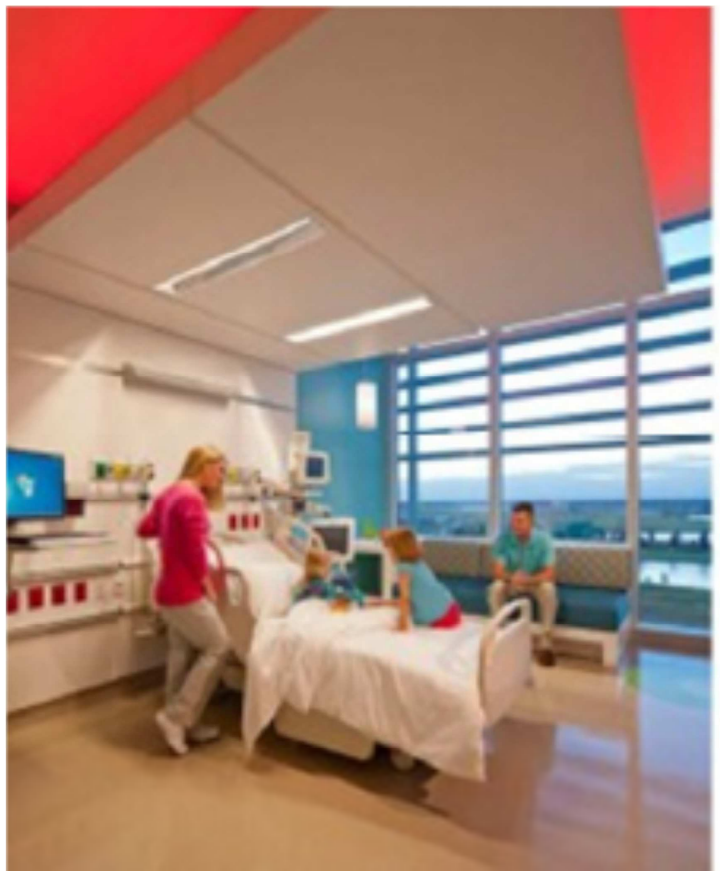

Fig. 4. Using energetic colors in composition with neutral colors in hospitalization room for children (Source: stanleybeamansears.com).

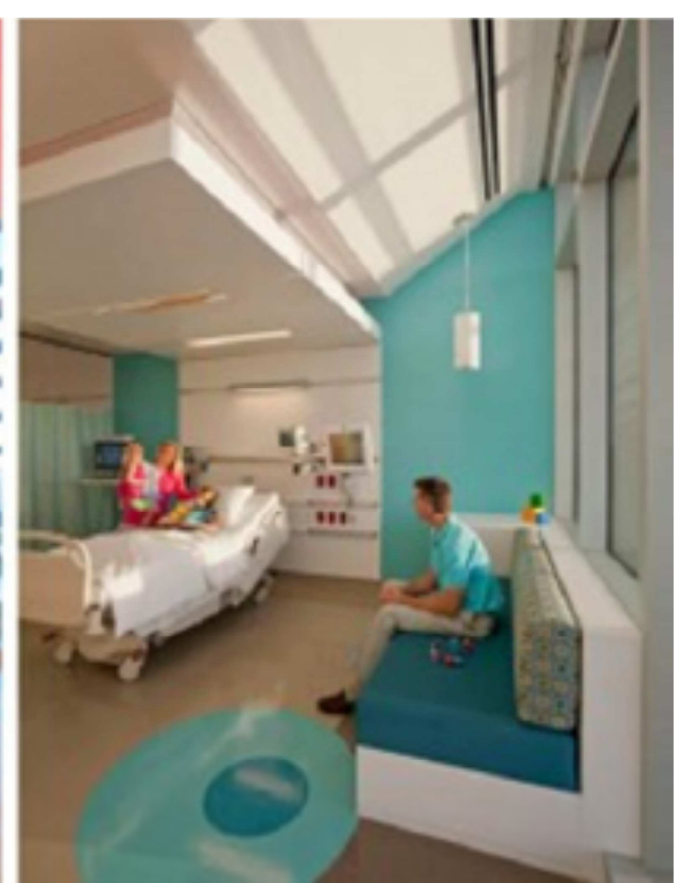

walls, ceiling and furniture) may play crucial role in creating enthusiasm, eager, and capability of children for better perception of space. The criterion of architects in selection of color may not be only restricted to color, kind, and duration of them, but it should be based on children's perception of colors. Selection of bothering colors at large scale by architects in order to make children's space happy and attractive may usually lead to reverse result since such colors cause imbalance in children's eyes. Happy and bright colors that create excitation in children are the best color for painting. Some of ideal colors which are widely utilized in design of playing rooms for children include bright spectrum of blue, green, yellow, pink, and orange colors. Diversity of colors makes the space more exciting. Painting a wall by a bright color and using complementary colors in adjacent wall is one of these strategies (Shamgholi, 2011). 


\section{Conclusion}

Surely, attendance in a hospital may be unpleasant experience for anyone since even patient's companions may also suffer from presence in environment of hospital in addition to physical impairments and pains.

While the aforesaid concerns and pains will be combined by deficiencies and weakness in giving services and will be followed by the possible worst experience for the patient and his/ her companions. For this reason, it is tried in most of advanced countries to design and build hospital environment based on the latest daily technologies to guarantee mental comfort for the patients. Investigations of medical sciences have also emphasized for several times in direct relationship among mental comfort of patient with advancement of treatment process more than ever.

These cases are highly important in relationship with children and infants further since the children should be treated and recovered in such an environment. As it explored in the present article, one can conclude that to what extent the color effect in hospital environmental graphic design may influence in child's mind and his/ her companions and contribute child to treat disease.

- Generally:

- Not using dark colors in children ward

- Application of happy and energetic colors such as redviolet-yellow-orange- in composition with neutral colors such as white, brown, and gray

- Using mild colors without eyesore for nursing station in pediatric ward including green- blue-pink in composition with neutral colors

- Employing energetic colors for the parts used by patient's companions due to requirement for recharge of energy such as orange-blue

- Using colors as symbol of purity and cleanness and ones shows the pollution immediately in healthcare spaces and operation rooms such as white- bright bluebright orange

- Therefore one can return relatively the lost comfort to child by employing these colors in environmental graphic design in medical spaces and reduce stress and fear caused by disease and therapeutic environments.

\section{References}

[1] Itten, Johannes (2005), Itten color elements, transl. Zhalehdoot Behrooz, Efaf Pub, Tehran
[2] Hosseini, Behshid, Sadeghi, Aida (2013), Importance of color in hospital care units from psychological perspective, Book of secure hospital

[3] Davie, Sadka (2014), transl. Al-E-Yasin Mohammad Reza, Tehran: Hamoon Pub

[4] Riazi, Zahra (2013), Effects of color and form in kindergarten environmental graphic design, Art book letter

[5] Sayari, Ali Akbar (2002), Health appearance in the world and Iran, Ministry of Health, Treatment and Medical Training, Tehran

[6] Abkar, ali, zamani, sajad (2013), \The effect of light and color in interior design in terms of quality therapeutic healing environment, The third National Conference of Interior Design and Decoration.

[7] Shamgholi, Gholamreza (2011), Basic concepts in hospital architectural design, Soroush Pub, Tehran

[8] Copper, Maimy (2014), Knowledge and psychology of colors for progress in personal and occupational life, transl. Maleki Amir Hossein, Tehran: Aria Gohar Pub

[9] Gorji Mahlebani, Yousef, Saleh Ahangar, Mojgan (2014), The color effect in design of hospitalization ward at hospitals, Scientific- Research Journal of Iranian Scientific Association of Architecture and Urbanism

[10] Mirzakhani, Elham \& Parviz (2012), Analysis on effect of group training of mothers on increasing creativity of children by focus on analytical method of mutual behavior, Quarterly of innovation and creativity in Human Sciences (2)

[11] Murphy (2014), Full collection of Murphy's Laws, transl. Saleh Ramsarai Ibrahim, Tehran: Moein Pub

[12] Wills, Pauline (2013) Chromotherapy, transl. Faraji Marjan, Tehran: Dorsa Pub

[13] Paavo, Airola (2008), Handbook of natural healing as way of achieving of full health, transl. Ali Akbar Radpoor, Tehran: Sales Pub

[14] Arthur, P., \& Passini, R. (1992), "Way-finding: People, signs and architecture". New York: McGraw-Hill Inc

[15] Baskaya, A. Wilson, C. \& Ozcan, Y. (2004), "Wayfinding in a unfamiliar environment. Different spatial

[16] Settings of two polyclinics. Environment and Behavior, 867839), 6 (36. Bently, J. (Author) and Turner, G. P. A. (Author) (1997). Introduction to Paint Chemistry and Principles of Paint Technology. Unk. ISBN-72320-412-4

[17] Talbert, Rodger (2007). Paint Technology Handbook. Grand Rapids, Michigan, USA. ISBN 1 